\title{
BMJ Global Health Cost and cost-effectiveness of childhood cancer treatment in low-income and middle-income countries: a systematic review
}

\author{
Alastair Fung, ${ }^{1}$ Susan Horton, ${ }^{2}$ Veda Zabih, ${ }^{3}$ Avram Denburg, ${ }^{3,4}$ Sumit Gupta ${ }^{3,4}$
}

To cite: Fung A, Horton S, Zabih V, et al. Cost and costeffectiveness of childhood cancer treatment in lowincome and middle-income countries: a systematic review. BMJ Global Health 2019;4:e01825. doi:10.1136/ bmjgh-2019-001825

Handling editor Soumyadeep Bhaumik

- Additional material is published online only. To view please visit the journal online (http://dx.doi.org/10.1136/ bmjgh-2019-001825).

Received 6 July 2019 Revised 20 September 2019 Accepted 12 0ctober 2019

Check for updates

(c) Author(s) (or their employer(s)) 2019. Re-use permitted under CC BY-NC. No commercial re-use. See rights and permissions. Published by BMJ

${ }^{1}$ Pediatrics and Child Health, Winnipeg Children's Hospital, Winnipeg, Manitoba, Canada ${ }^{2}$ School of Public Health and Health Systems, University of Waterloo, Waterloo, Ontario, Canada

${ }^{3}$ Child Health and Evaluative Sciences, Hospital for Sick Children, Toronto, Ontario, Canada

${ }^{4}$ Division of Hematology/ Oncology, Hospital for Sick Children, Toronto, Ontario, Canada

Correspondence to

Dr Sumit Gupta;

sumit.gupta@sickkids.ca

\section{ABSTRACT}

Introduction A major barrier to improving childhood cancer survival is the perception that paediatric oncology services are too costly for low-income and middleincome country (LMIC) health systems. We conducted a systematic review to synthesise existing evidence on the costs and cost-effectiveness of treating childhood cancers in LMICs.

Methods We searched multiple databases from their inception to March 2019. All studies reporting costs or cost-effectiveness of treating any childhood cancer in an LMIC were included. We appraised included articles using the Consolidated Health Economic Evaluation Reporting Standards (CHEERS) checklist. Where possible, we extracted or calculated the cost per disability-adjusted life year (DALY) averted using reported survival and countryspecific life expectancy. Cost/DALY averted was compared with per capita gross domestic product (GDP) as per WHOChoosing Interventions that are Cost-Effective guidelines to determine cost-effectiveness.

Results Of 2802 studies identified, 30 met inclusion criteria. Studies represented 22 countries and nine different malignancies. The most commonly studied cancers were acute lymphoblastic leukaemia $(n=10)$, Burkitt lymphoma $(n=4)$ and Wilms tumour $(n=3)$. The median CHEERS checklist score was 18 of 24. Many studies omitted key cost inputs. Notably, only 11 studies included healthcare worker salaries. Cost/DALY averted was extracted or calculated for 12 studies and ranged from US\$22 to US\$4475, although the lower-end costs were primarily from studies that omitted key cost components. In all 12, cost/DALY averted through treatment was substantially less than country per capita GDP, and therefore considered very cost-effective.

Conclusion Many included studies did not account for key cost inputs, thus underestimating true treatment costs. Costs/DALY averted were nonetheless substantially lower than per capita GDP, suggesting that even if all relevant inputs are included, LMIC childhood cancer treatment is consistently very cost-effective. While additional rigorous economic evaluations are required, our results can inform the development of LMIC national childhood cancer strategies.

\section{Key questions}

What is already known?

- There is a paucity of data on the full economic costs and cost-effectiveness of childhood cancer treatment in low-income and middle-income countries (LMICs).

- A systematic review in 2013 identified and assessed economic evaluations for paediatric cancer treatment but only included studies from high-income countries.

What are the new findings?

- This study is the first systematic review to synthesise the existing evidence on the cost and costeffectiveness of a range of paediatric oncology treatments in LMICs.

- Although many included studies did not account for key cost inputs, costs/disability-adjusted life year averted were substantially lower than per capita gross domestic product, suggesting that even if all relevant inputs are included, LMIC childhood cance treatment is consistently very cost-effective.

What do the new findings imply?

- Future research should include more rigorous economic evaluations of paediatric oncology services in LMICs.

- Our results demonstrating that childhood cance treatment is generally very cost-effective can inform the prioritisation of childhood cancers on global health agendas and the development of national childhood cancer strategies in LMICs.

\section{INTRODUCTION}

In 2018 , there were 18.1 million new cases of cancer and 9.5 million cancer-related deaths worldwide. ${ }^{1}$ While the overall incidence of cancer is lower in low-income and middleincome countries (LMICs) compared with high-income countries (HICs), approximately $70 \%$ of cancer deaths occur in LMICs. ${ }^{12}$ The global cancer burden is expected to increase to 23.6 million new cases per year by $2030 .^{3}$ 
The incidence of childhood cancer has been increasing over time with a growing proportion of this burden falling on LMICs. In 2008, there were 175058 new cases of childhood cancer worldwide, though the true estimates were likely much higher due to ongoing issues with under-diagnosis and under-registration. ${ }^{4} \mathrm{~A}$ recent simulation-based study estimated that 397000 new cases of childhood cancer occurred globally in 2015, including both diagnosed and undiagnosed cases. ${ }^{5}$ An analysis of the Global Burden of Disease study estimated a global incidence of 416500 new cases of childhood cancer in $2017{ }^{6}$ Given that LMIC populations have higher relative proportions of children than HICs, more than $80 \%$ of childhood cancers actually occur in LMICs. ${ }^{4}$ In 2017 , childhood cancers contributed to 11.5 million disabilityadjusted life years (DALYs) globally with $82 \%$ of this burden affecting low, low-middle and middle Sociodemographic Index countries. ${ }^{6}$

Among children aged 0-14 in LMICs, deaths related to communicable diseases continue to exceed cancerrelated deaths. ${ }^{4}$ LMIC governments have therefore generally prioritised healthcare resource allocation to communicable rather than non-communicable diseases (NCDs) such as cancer. ${ }^{4}$ However, as their economies develop, LMICs undergo epidemiological transition, with a decreasing burden of communicable diseases and an increase in mortality from cancer and other NCDs. ${ }^{4}$ Indeed from 1990 to 2011, among 106 LMICs, 80\% of the reduction in childhood mortality was attributed to decreased deaths from communicable diseases. ${ }^{8}$ NCDs including cancer thus comprise a growing proportion of global childhood mortality. Indeed, from 2005 to 2016, cancer was among the top three causes of death in children aged 5-14 years in Mexico, Brazil and China. ${ }^{9}$

Survival rates among children diagnosed with cancer in HICs receiving modern multidisciplinary treatments exceed $80 \%{ }^{10}$ However, paediatric cancer survival rates in LMICs range from $10 \%$ to $50 \% .{ }^{11}$ Reasons for this survival gap include delayed presentation, lack of efficient referral pathways, treatment abandonment, increased treatment toxicities, and poor access to chemotherapy. ${ }^{411} 12$ A recent survey of lead clinicians from nine LMICs noted poor access to chemotherapy for leukaemias, lymphomas, Wilms tumour and retinoblastoma. ${ }^{12}$ In addition to medical treatments, over $90 \%$ of the population of low-income countries (LICs) lack access to radiation therapy. ${ }^{13}$ In an effort to surmount these challenges, a number of bilateral and regional collaborative childhood cancer initiatives have been developed in LMICs. ${ }^{11}$ These efforts, including partnerships with paediatric cancer centres in HICs, promote capacity building, education of healthcare personnel, implementation of treatment strategies, and establishment of research programmes adapted to local capacity and needs. ${ }^{11}$ However, recognition of the limits of such initiatives in terms of health system integration and sustainability has prompted increasing emphasis on the importance of national childhood cancer strategies, and evidence to support governmental priority-setting accordingly. ${ }^{14}$

A major barrier to developing national childhood cancer strategies and improving childhood cancer survival is the perception that cancer services, including paediatric oncology services, are too costly for LMIC health systems. ${ }^{15}$ Recent evidence challenges this perception ${ }^{16-18}$ but a paucity of data on the full economic costs and cost-effectiveness of childhood cancer treatment in LMICs remains. A recent systematic review evaluated treatment costs in LMICs but focused specifically on acute lymphoblastic leukaemia (ALL) ${ }^{19}$ Another systematic review identified and assessed economic evaluations for paediatric cancer treatment but only included studies from HICs. ${ }^{20}$

Building on the Global NCD Action Plan (2013-2030) and the World Cancer Declaration 2013, the 2017 Cancer Resolution reaffirmed cancer control as a global priority and provided a framework for countries to strengthen their cancer services. ${ }^{21}$ In September 2018, the WHO launched a new endeavour, the WHO Global Initiative for Childhood Cancer, with the goal of achieving a global survival rate of at least $60 \%$ for children with cancer by $2030 .^{22}$ In light of these global goals, synthesising data from multiple economic analyses to evaluate the costeffectiveness of childhood cancer treatment in LMICs can provide evidence to inform policy agenda-setting, health system priority-setting, and the development of national childhood cancer strategies in LMICs.

The objective of this study was therefore to undertake a systematic review of existing economic evaluations of childhood cancer treatment in LMICs and to assess their methodological rigour. We also aimed to compile and summarise the costs and cost-effectiveness of treating different types of childhood cancers in various LMICs.

\section{METHODS}

\section{Search strategy and selection criteria}

This study followed the Preferred Reporting Items for Systematic Reviews and Meta-Analyses checklist (online supplementary appendix A). Study inclusion criteria were: (1) paediatric participants ( $<18$ years of age); (2) analysis of the cost, cost-effectiveness, cost-utility, or costbenefit of any medical, surgical, or radiation therapy interventions to treat any childhood cancer listed in the International Classification of Childhood Cancer, third edition (ICCC-3) ${ }^{23}$; (3) measurement of economic costs, cost per life saved, cost-effectiveness ratios, cost per life year (LY) or quality-adjusted life year (QALY) gained, or cost per DALY averted; and (4) conducted in LMICs as defined by the World Bank. ${ }^{24}$ No restrictions were placed on language or dates published.

We excluded any study that assessed diagnostic modalities, supportive care, allied health, or palliative care in isolation. Studies conducted in HICs, review articles, conference abstracts, case reports, commentaries and editorials were also excluded. 
We searched EMBASE, Medline, Cochrane library and Web of Science for all relevant articles published from their inception to 23 March 2019. Search strategy concepts included 'cancer', 'child', 'developing countries', 'costs and cost analysis', 'cost-effectiveness analysis' and 'healthcare costs'. We scanned the reference lists of eligible full-text articles to search for potential articles not identified in the original database search. The full electronic search strategy for Medline can be found in online supplementary appendix B.

\section{Data analysis}

Two reviewers (AF and VZ) independently screened all abstracts. Both reviewers then retrieved all studies potentially meeting inclusion criteria in full and assessed the eligibility of each full-text article. Disparities between reviewers were resolved by discussion with the research group. The kappa measure of agreement was calculated between the two reviewers.

All eligible full-text articles were either in English or Spanish. Both reviewers extracted data from all included English studies using a standardised form (online supplementary appendix C). Two studies were in Spanish and SG extracted the data from these studies. Discrepancies between reviewers were discussed and resolved by consensus. Information collected included first author and year of publication, country and study setting, study design, cancer diagnosis, analytical perspective of the economic evaluation, inputs used in the cost analysis, data sources, survival outcomes, and cost, cost-effectiveness, and cost-utility outcomes.

We converted all cost outcomes to 2018 USD using the International Monetary Fund Consumer Price Indices and exchange rates. ${ }^{25}$ We used the exchange rate of the original year to convert local currencies to USD. If the article did not specify the original currency year, we used the last year of data collection. If a study did not report cost-effectiveness or cost-utility outcomes but reported survival outcomes and cost outcomes, we calculated the cost per DALY averted using a discount rate of $3 \%$ on future years survived as recommended by the WHO-Choosing Interventions that are Cost-Effective (CHOICE) guidelines. ${ }^{26}$ We assumed a mean age at diagnosis of 6 years based on data from the Hospital Nacional de Ninos Benjamin Blum in El Salvador which, to our knowledge, was the first study to report estimates of the cost of maintaining a paediatric cancer programme in an LMIC. ${ }^{18}$ If a study reported 1-year but not 5-year overall survival, we used literature from comparable settings to estimate 5 -year survival. ${ }^{27}$ We used event-free survival in our calculations if overall survival was not reported.

We used the WHO-CHOICE thresholds to determine cost-effectiveness. ${ }^{26}$ According to these criteria, interventions with a cost per DALY averted less than country per capita gross domestic product (GDP) are considered very cost-effective and those with a cost per DALY averted less than three times country per capita GDP are cost-effective.
Both reviewers appraised the methodological quality of the included studies using the Consolidated Health Economic Evaluation Reporting Standards (CHEERS) checklist (online supplementary appendix E). ${ }^{28}$ This checklist identifies 24 key items and methodological characteristics recommended by the International Society for Pharmacoeconomics and Outcomes Research (ISPOR) to be reported in economic evaluations. Each item was given a score of one point and two-part items were divided into two half-points. Discordance in quality assessment was resolved by discussion.

Although the CHEERS checklist provides a validated scoring system for overall methodological study quality, it does not include an appraisal of the number of childhood cancer-specific inputs included in a given article's cost analysis. Therefore, in addition to the CHEERS checklist score, we also created a ranking system to denote the comprehensiveness of the inputs included in cost analyses. Missing inputs affect the accuracy of the cost and cost-effectiveness outcomes. More comprehensive studies provide better estimates of total costs whereas less comprehensive studies underestimate total costs. We specified 14 inputs in our data extraction sheet which we felt were important to include in a comprehensive cost analysis of paediatric cancer treatment (online supplementary appendix D). Studies that included 10 or more inputs were ranked as 'Comprehensive', studies with five to nine inputs were ranked as 'Medium', and studies with less than five inputs were ranked as 'Low'.

\section{Patient and public involvement and ethics approval}

We did not involve patients or the public in the design, conduct, or reporting of this study. Given that we used published articles for this systematic review, Research Ethics Board approval was not required.

\section{RESULTS}

Our systematic literature search identified 2802 articles. After adjusting for duplicates, 2001 studies remained. Of these, 30 studies met full inclusion criteria. ${ }^{16-18} 29-55$ The kappa measure of agreement between the two reviewers was 0.71 (95\% CI 0.55 to 0.86 ). The flow diagram for study selection is shown in figure 1 . The characteristics of the 30 included articles are summarised in table 1 . The included studies were published between 2003 and 2019. Studies represented 22 countries across three continents (figure 2). The cancers investigated included ALL $(\mathrm{n}=10)$, Burkitt lymphoma $(\mathrm{n}=4)$, Wilms tumour $(\mathrm{n}=3)$, retinoblastoma $(n=3)$, Hodgkin lymphoma $(n=1)$, acute myeloid leukaemia (AML) $(\mathrm{n}=1)$, acute promyelocytic leukaemia (APL) $(n=1)$, osteosarcoma $(n=1)$ and extracranial germ cell tumours $(n=1)$. Five studies investigated multiple childhood cancers.

Table 1 shows the CHEERS checklist score for each study. The median CHEERS checklist score was 18 of 24 with an IQR of 6 . Omitting or not specifying key inputs into the cost analysis was a common methodological error, 


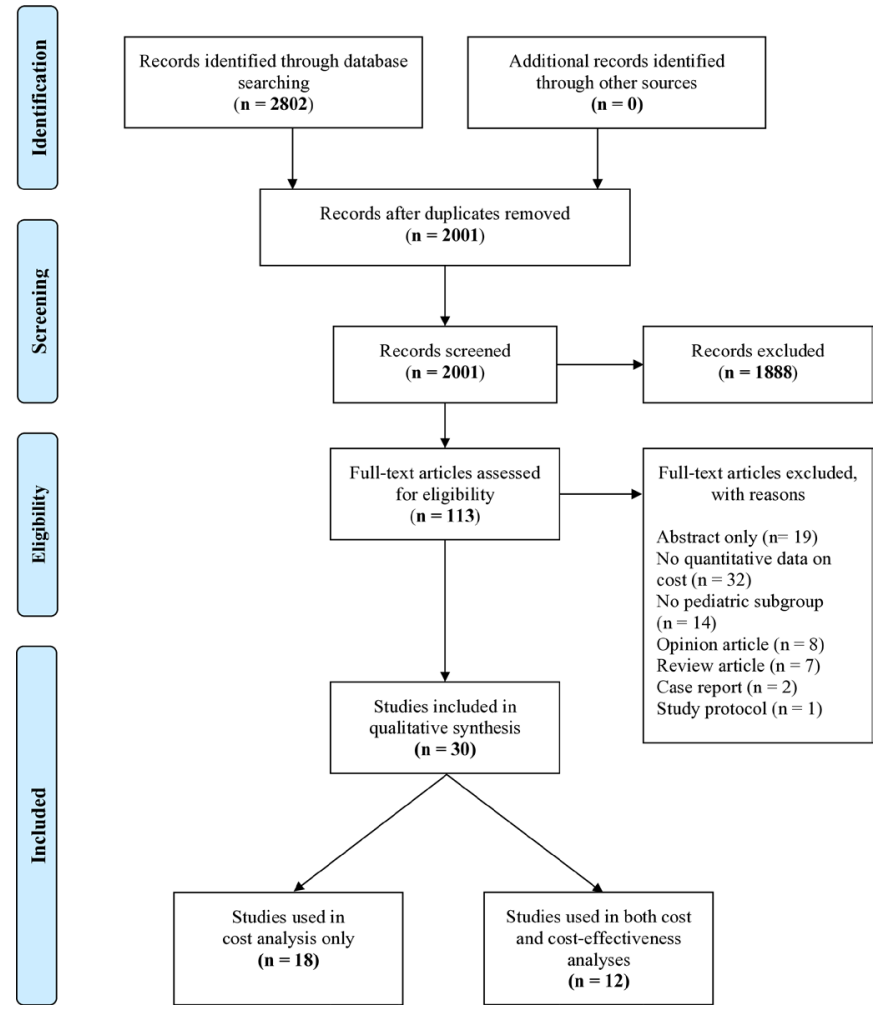

Figure 1 Flow diagram for study selection.

thus underestimating total costs. Regarding the comprehensiveness of included inputs, seven studies ranked as 'Comprehensive', eight studies ranked as 'Medium', and 14 studies ranked as 'Low'. Healthcare worker salaries were included in only 11 studies. Other significant costs such as patient accommodations and facility administration were included in only 15 studies and three studies, respectively (online supplementary appendix D).

Of the 30 included studies, cost per DALY averted could be extracted or calculated for 12 studies and ranged from US $\$ 22$ to US $\$ 4475$ (table 2). The cancer that was most cost-effective to treat was Burkitt lymphoma; if this cancer was excluded, along with the studies ranked as 'Low' in comprehensiveness of costs included, the range was US $\$ 800$ to US $\$ 4475$. Figure 3 shows the cost per DALY averted through treatment of a range of cancers relative to country per capita GDP for studies that most closely estimated true costs ('Comprehensive' and 'Medium' ranked studies). Among these studies, the ratio of cost per DALY averted to per capita GDP ranged from 0.09 to 0.8. Cost per DALY averted through treatment was substantially less than country per capita GDP, and therefore considered very cost-effective per WHO-CHOICE thresholds.

\section{DISCUSSION}

Our findings summarise the existing evidence on costs and cost-effectiveness of various paediatric cancer treatments in LMICs. Where available, the cost per DALY averted associated with childhood cancer treatment was less than country per capita GDP, thus meeting criteria for being very cost-effective as per WHO-CHOICE guidelines.

GDP-based cost-effectiveness thresholds are the subject of ongoing debate and suggested cut-offs vary widely among experts. Some scholars have argued that routinely used cost-effectiveness thresholds such as WHO-CHOICE are too high because they do not reflect health opportunity cost-that is, they do not account for health losses that occur because other interventions cannot be provided. ${ }^{56}$ The upper bounds of opportunity-cost-based cost-effectiveness thresholds have been estimated to be 0.51 and 0.71 times per capita GDP for LMICs, respectively. ${ }^{56}$ On the other hand, recent investment cases involving WHO health economists regard the total value of a life year across economic and social components to be 1.5 times GDP per capita, while the World Bank estimates the value of a life year at 1.4-4.2 times GDP per capita. $^{57}$

Among the included studies we ranked as 'Comprehensive' or 'Medium', using the more conservative opportunity-cost-based thresholds, treatment of all childhood cancers in El Salvador and Ghana, ALL in Mexico, ALL in China, and Burkitt lymphoma in Uganda would remain cost-effective while treatment of ALL in Iran would no longer be cost-effective.

It is also important to note that the cost-effectiveness of an intervention may change over time. As shown in table 2, when comparing the three studies ranked as 'Comprehensive', as country GDP per capita increased, costs per DALY averted also increased. This parallel increase can be partly attributed to non-tradable costs such as healthcare personnel salaries and patient accommodations that increase as a country's income level increases. In addition, as newer treatments or improved supportive care become available, survival rates improve over time, thereby impacting cost-effectiveness.

One systematic review identified and described economic evaluations of paediatric cancer treatment in HICs. ${ }^{20}$ Of the 40 studies identified in this review, 29 were supportive care studies (eg, growth colony stimulating factor) and 11 assessed tumour-directed therapies. The 11 tumour-directed therapy studies either investigated advanced interventions rarely described in LMICs such as stem cell transplant and radiation therapy or did not report cost-effectiveness as cost per DALY averted which limits comparison with results from our review.

Although global health agendas have generally prioritised treatment of communicable diseases, the prevalence of NCDs such as childhood cancer is rising in LMICs. ${ }^{4}$ Our study found that the cost per DALY averted for treatment of various paediatric cancers in LMICs ranged from US $\$ 22$ to US $\$ 4475$, or US $\$ 800$ to US $\$ 4475$ when excluding Burkitt lymphoma and studies ranked 'Low' in comprehensiveness of costs. These figures are comparable to the cost per DALY averted of other widely accepted public health strategies for paediatric 


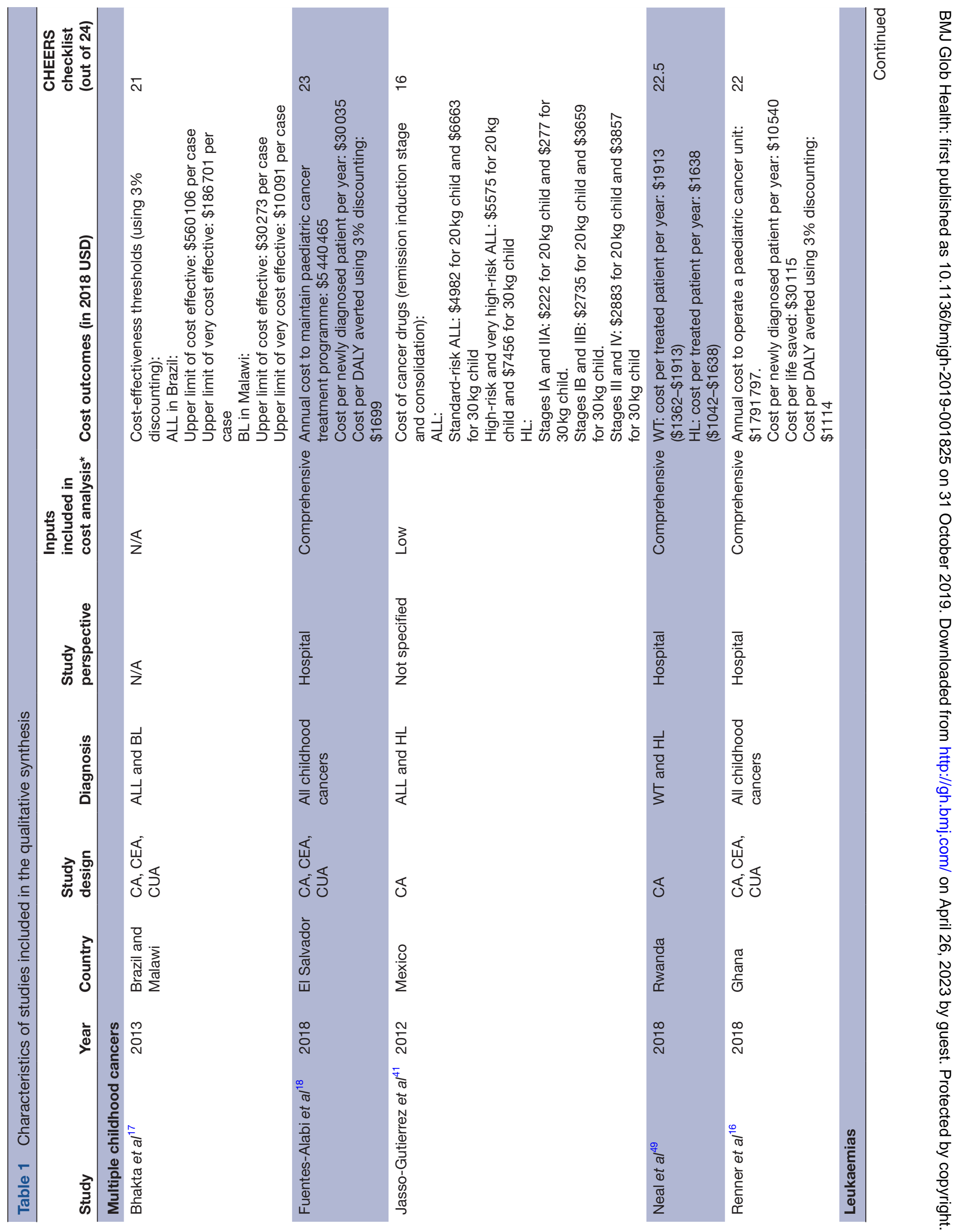




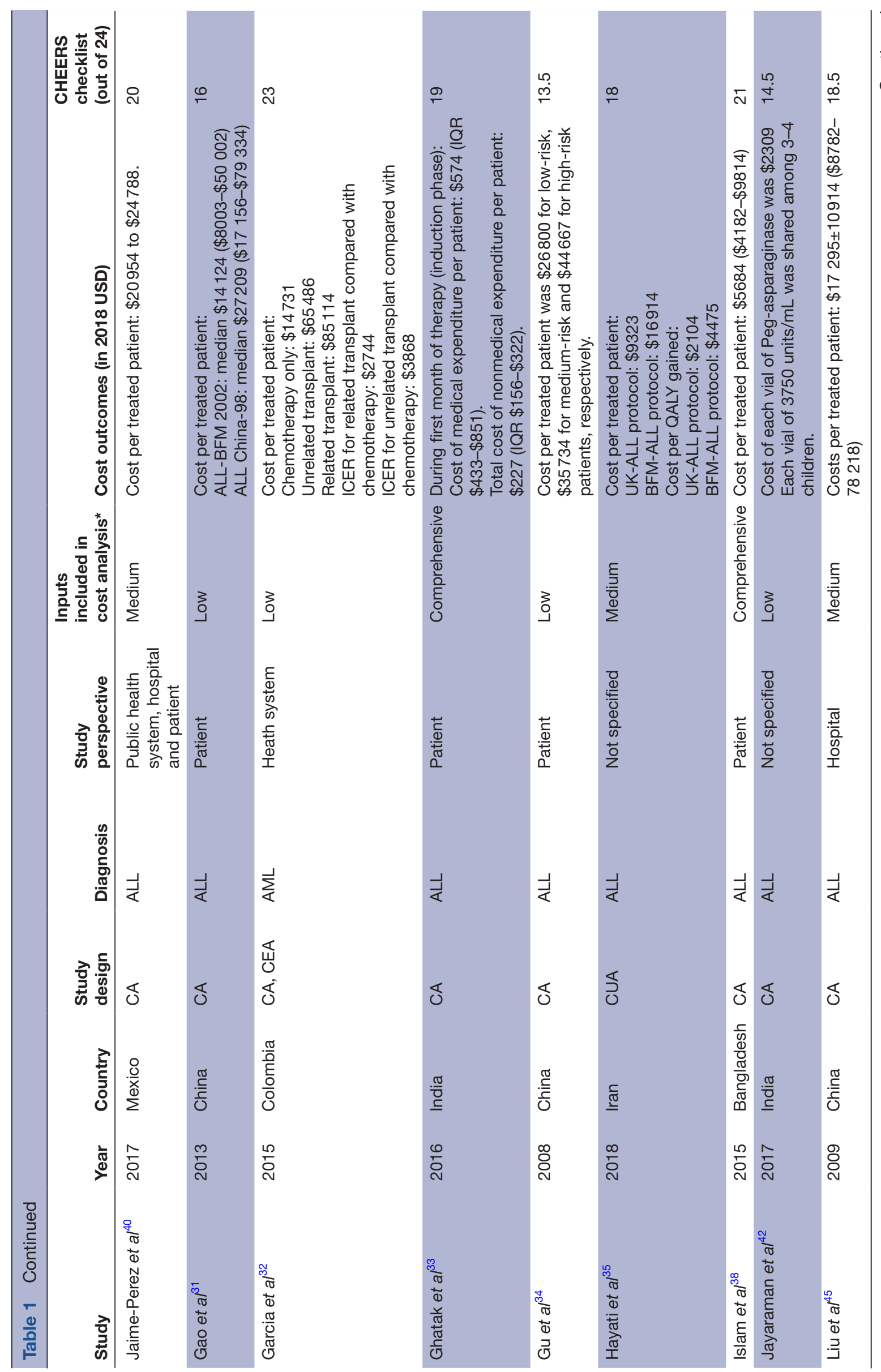



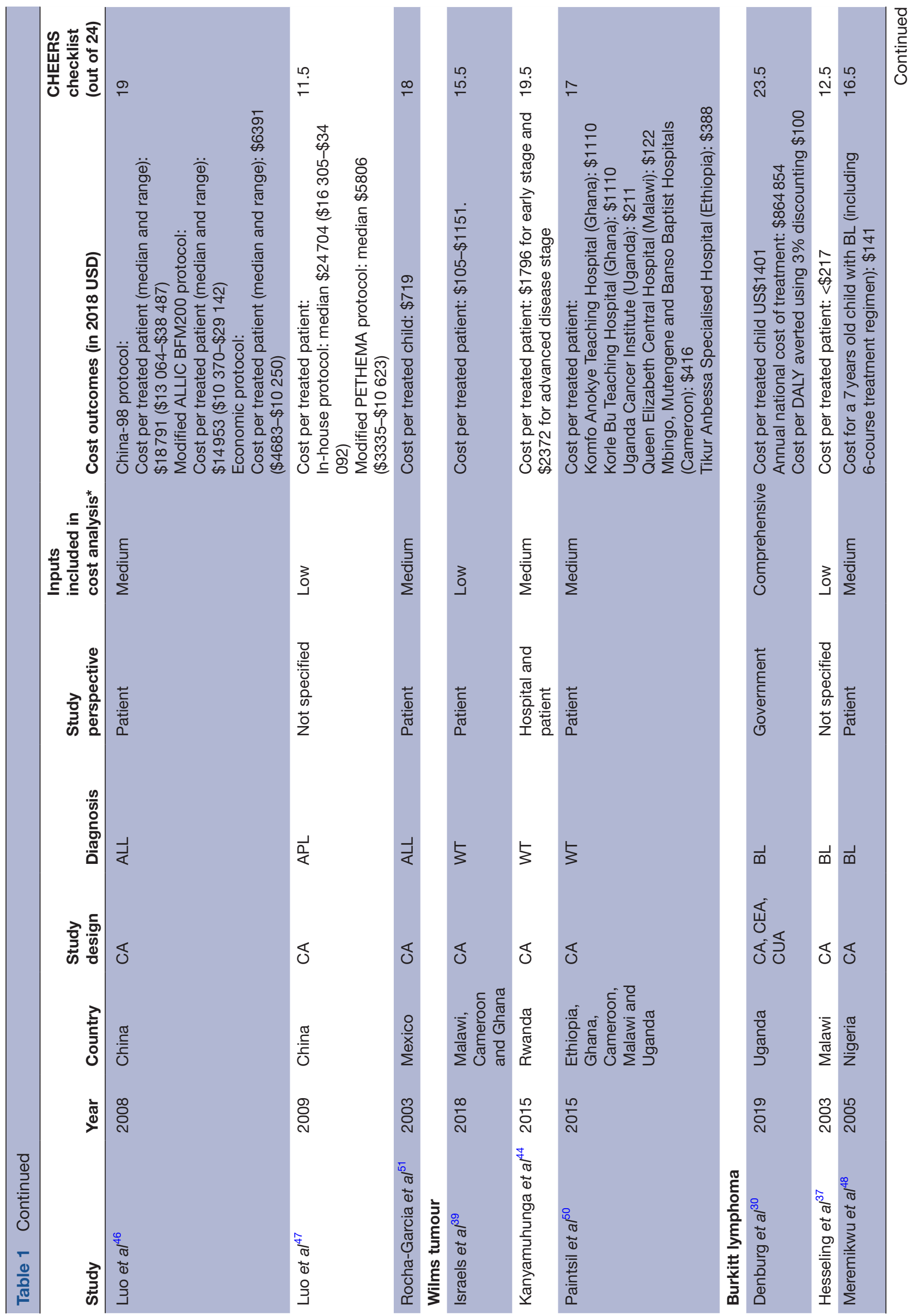

$\vec{m} \quad \vec{m} \vec{m}$

嵌

엉 ठ

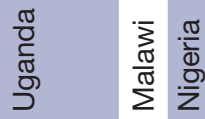

유 ஜ융

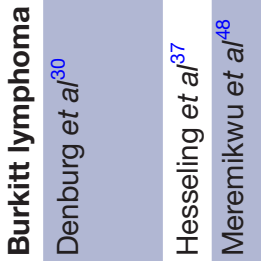




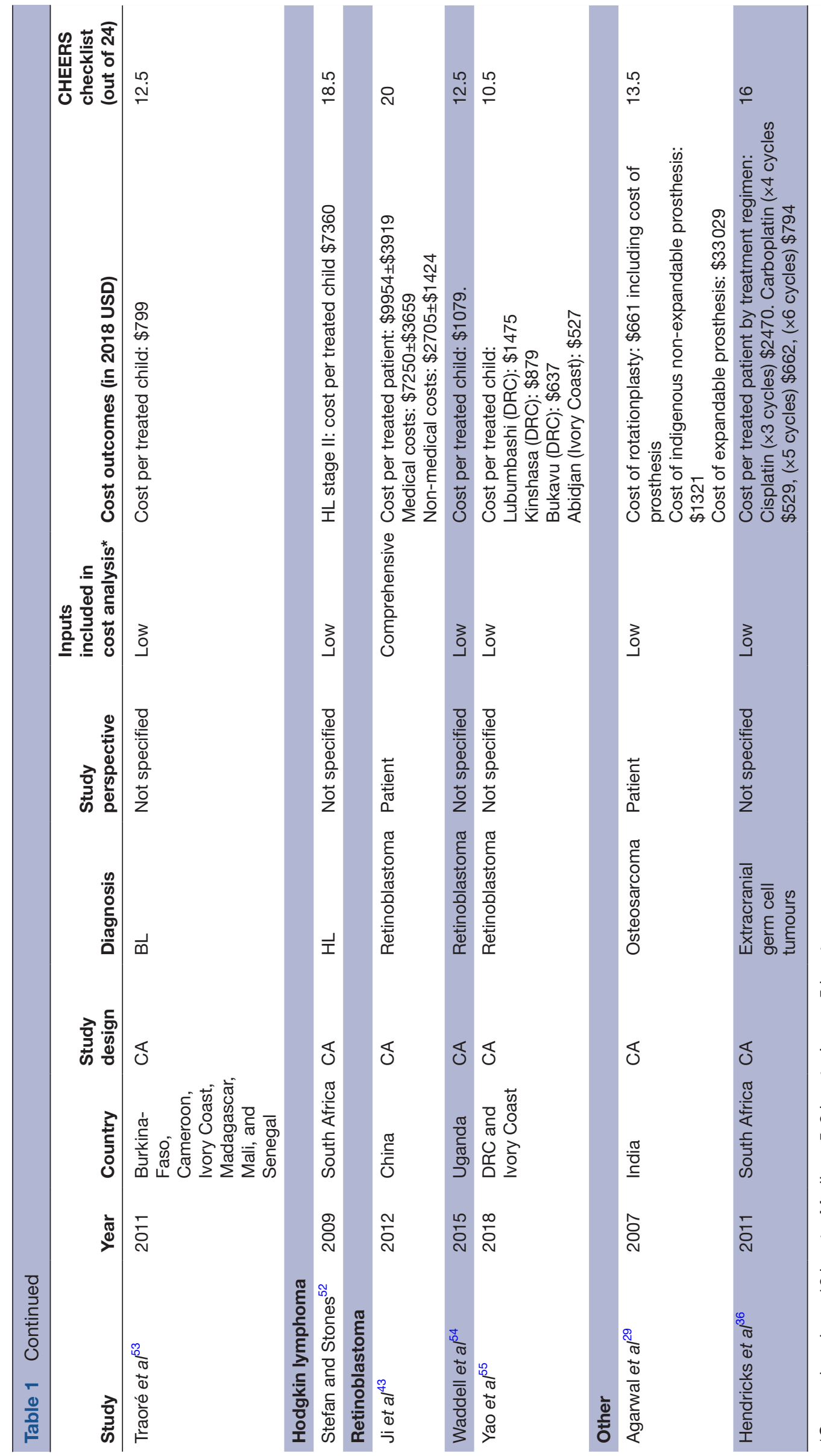

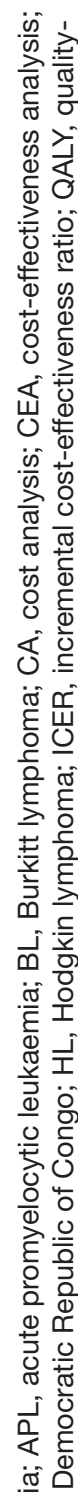




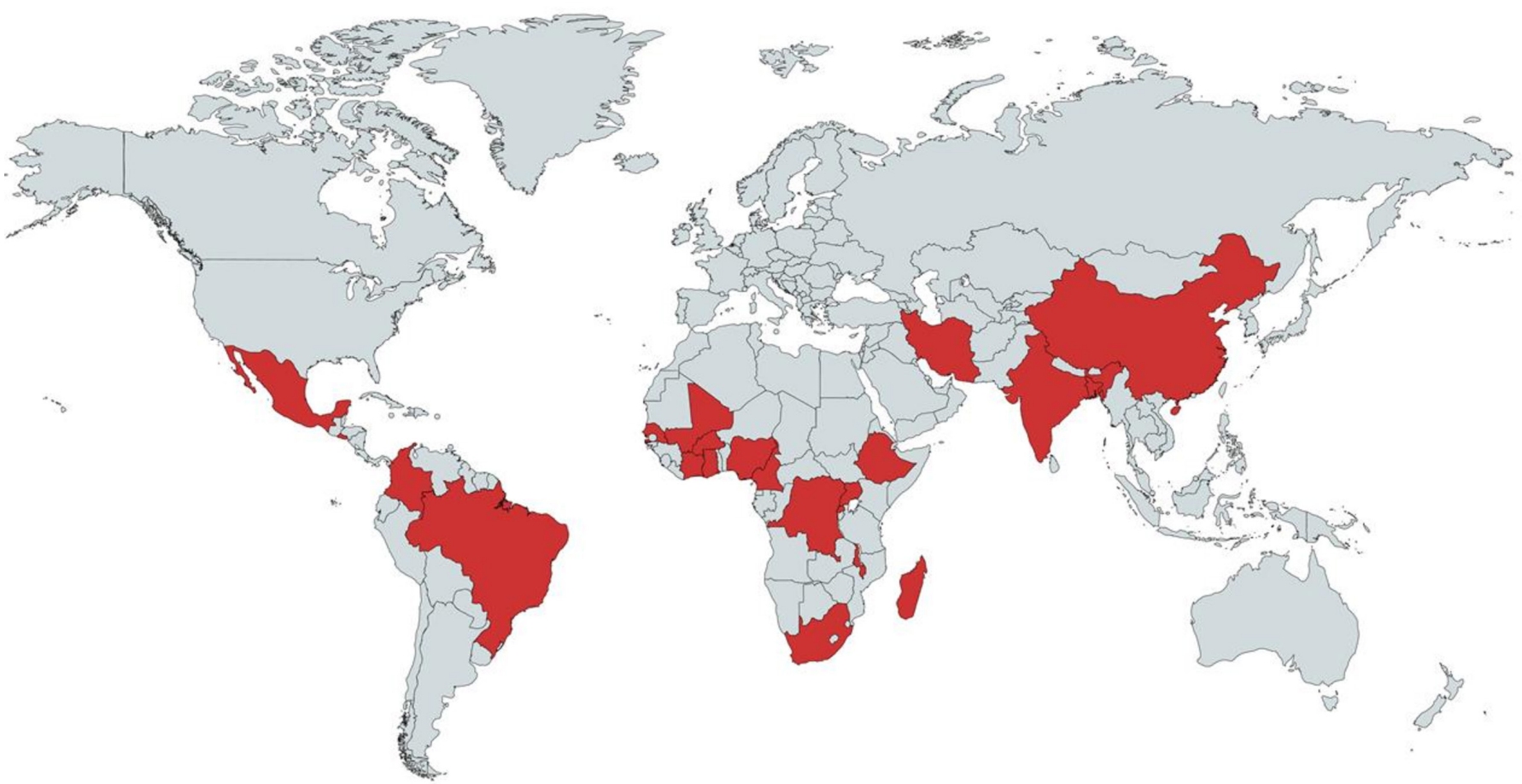

Figure 2 Countries represented in included studies.

communicable diseases such as pneumococcus, rubella and polio vaccines (2018 US\$1094 to US\$3281). ${ }^{58}$

The costs per DALY averted of treating childhood cancers in LMICs is also comparable to that of screening and treatment of breast cancer in LMICs (2018 US\$2010-US\$3913) and prevention of cervical cancer via human papilloma virus (HPV) vaccination (2018 US\$184-US\$5,652). ${ }^{59}$ There has been significant progress in funding and prioritisation of women's cancers in LMICs, most notably for breast and cervical cancers. Similar efforts to prioritise childhood cancer in global health have begun with the recent WHO Global Initiative for Childhood Cancer. Through this Initiative, the WHO will support governments in expanding childhood cancer services and integrating childhood cancer into national strategies and health insurance packages. ${ }^{22}$ Our findings demonstrate that these goals can be achieved cost-effectively.

Developing national childhood cancer strategies may require consideration of variations in incidence of different types of childhood cancer across LMICs. Of the 366600 new cases of childhood cancer estimated to have occurred in LMICs in 2015, the most common childhood cancer globally was ALL followed by non-Hodgkin's lymphoma, Wilms tumour, Burkitt lymphoma and retinoblastoma. ${ }^{5}$ ALL was the most common cancer in most regions of the world except for sub-Saharan Africa. ${ }^{5}$ However, the overall incidence of childhood cancer was more than five times higher in Africa compared with Europe and North America together due to increased incidence of types of cancer other than ALL. ${ }^{5}$ In western Africa, there was a significantly higher burden of lymphomas, retinoblastomas and renal tumours, including $60 \%$ of the global incidence of Burkitt lymphoma. South-central Asia had the highest incidence of ALL, AML, Hodgkin lymphoma, neuroblastoma and central nervous system (CNS) tumours. ${ }^{5}$

Moreover, as shown in tables 1 and 2, cost and costeffectiveness of treatment also vary by type of childhood cancer. One can assume that treatment costs would vary directly with treatment complexity including duration of chemotherapy, need for surgery, and need for supportive care. Treatment complexity is generally lower for malignancies requiring chemotherapy-only regimens of short duration such as Burkitt lymphoma and most lymphomas. Cancers requiring longer chemotherapy duration, such as ALL, or requiring surgery, such as Wilms tumour and retinoblastoma, are more complex, and cancers requiring highly intricate surgery, such as CNS tumours, or very highly intensive and prolonged chemotherapy, such as metastatic neuroblastoma, are most complex. ${ }^{60}$ It is also important to consider that availability of treatments, such as surgery or radiotherapy, may be a more significant barrier than cost in LMICs.

Although an intervention may be cost-effective, it is not necessarily affordable to families. In one study assessing the financial burden of Burkitt lymphoma treatment in Nigeria, one quarter of families could not afford the cost of diagnostic tests. ${ }^{48}$ About one-fifth of the children did not receive chemotherapy because their families were unable to pay and one-third of children withdrew from treatment due to financial constraints. ${ }^{48}$ Another study evaluating the costs to families of ALL treatment in India found that families spend up to seven times their monthly income during the first month of therapy. ${ }^{33}$ For children with leukaemia in LMICs, treatment abandonment rates 


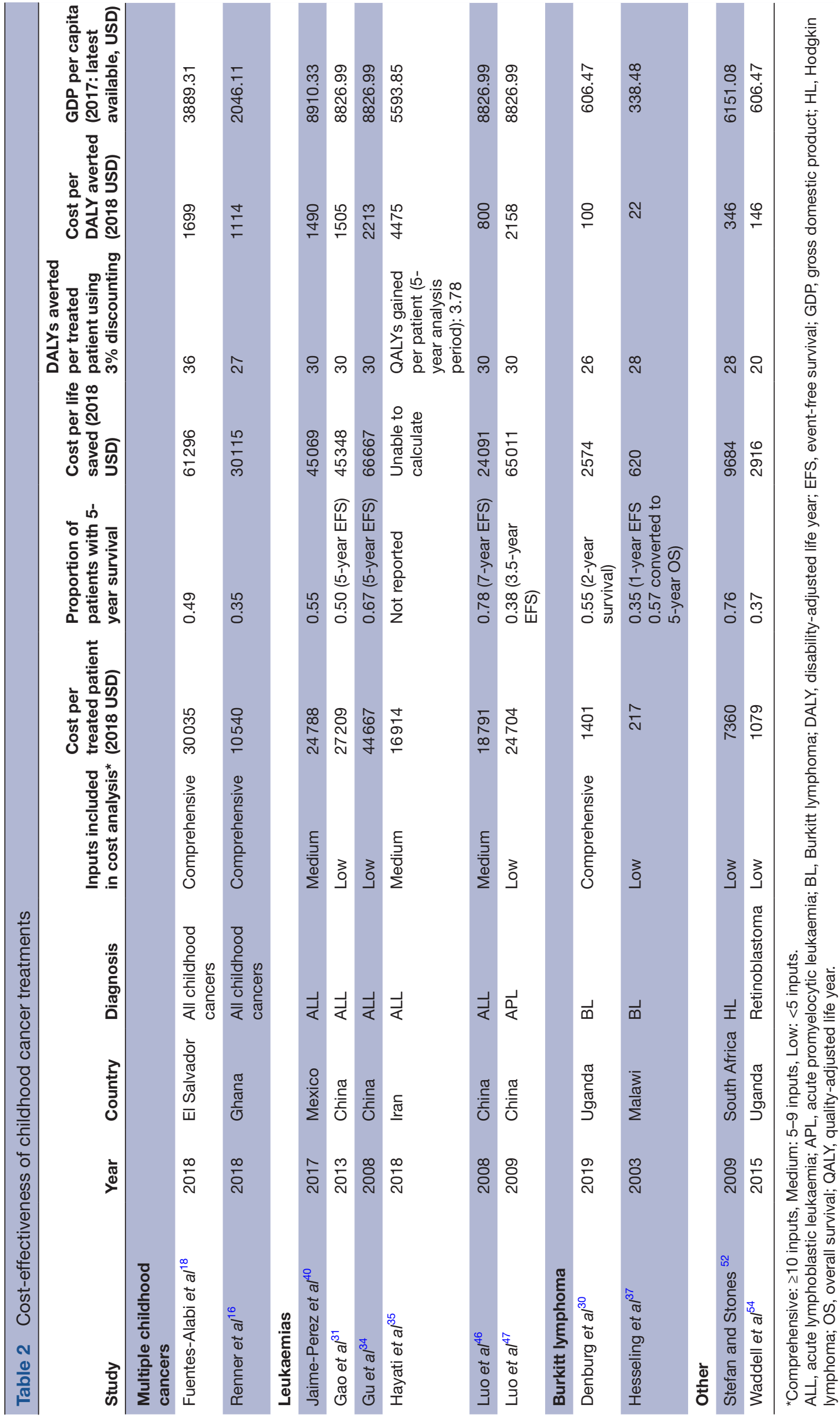

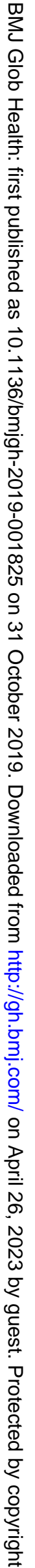




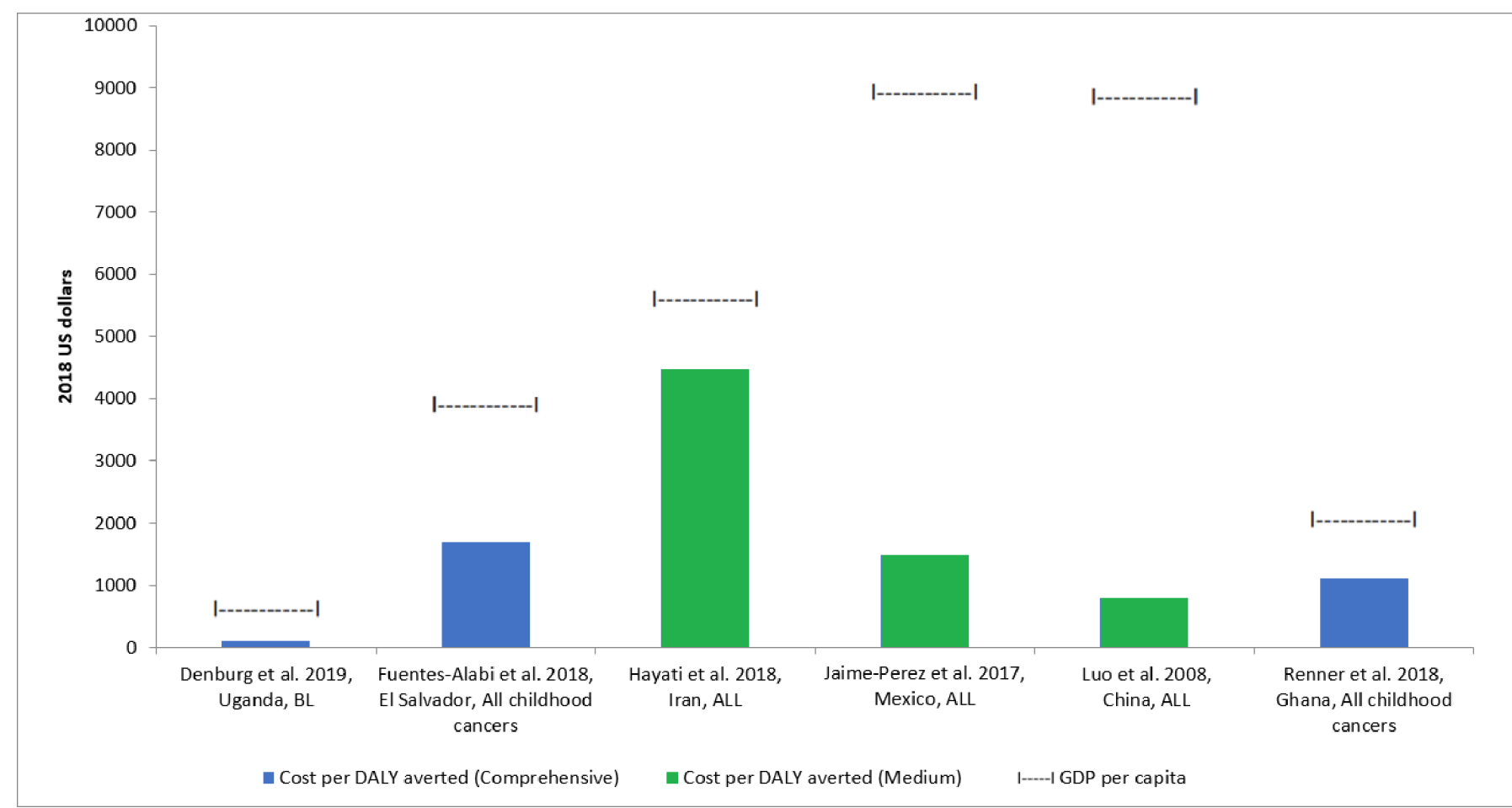

Figure 3 Cost-effectiveness of childhood cancer treatments*. ALL, acute lymphoblastic leukaemia; BL, Burkitt lymphoma; DALY, disability-adjusted life year; GDP, gross domestic product. *Only includes studies with extracted or calculated cost per DALY averted that were ranked as 'Comprehensive' or 'Medium' for number of inputs included in cost analysis.

as high as $74.5 \%$ have been described. ${ }^{61}$ Movements toward financial coverage of paediatric cancer treatment have begun in some LMICs including Tanzania, Mexico and China and are crucial to advancing affordability to families. ${ }^{62}$

Furthermore, the cost-effectiveness of an intervention does not necessarily imply that governments will prioritise a given issue including childhood cancer. In settings with limited resources, multiple factors such as justice, efficiency, political climate, economic growth and cultural values compete and contribute variably to shaping health system priorities. ${ }^{63}$ At times, social, cultural and political sentiments may prevail over cost-effectiveness or other purely economic factors in determining prioritisation of healthcare investments. Our findings can aid policymakers in considering childhood cancer treatment as a priority relative to other health interventions but cannot ultimately ensure allocation of policy attention and funding toward childhood cancer services in a given country's specific context.

The main limitation of this systematic review relates to the quality of the existing literature in this area. Few economic evaluations in our study were of high methodological rigour, as demonstrated by their CHEERS checklist scores. Several studies did not outline assumptions made in study design, describe the analytical perspective of the study, or report a discount rate, and only five studies included a sensitivity analysis, all of which contributed to lower CHEERS checklist scores. Clearly outlining assumptions and conducting sensitivity analyses help mitigate the uncertainties inherent in most economic evaluations. Future economic evaluations in this area should adhere to the CHEERS checklist, which consolidates previous economic evaluation guidelines and provides recommendations to optimise the design and reporting of economic evaluations in healthcare. Doing so will not only produce more accurate data to help guide policymakers, but will allow comparisons between studies and settings.

Another important limitation relates to costing methodology. Among the 'Comprehensive' studies, the most significant cost inputs were healthcare personnel, chemotherapy, surgery, patient accommodations and administration. Although each of the 30 included studies considered costs of chemotherapy and/or surgery, only 11 included healthcare personnel salaries, only 15 included patient accommodations, and only three included administration costs. Omission of key cost inputs, particularly hospital administration, which accounts for utilities, space, human resource managers, and patient record and cancer registry staff, significantly underestimates total cost. ${ }^{64}$ For studies investigating ALL, costs per treated child ranged from US $\$ 801$ to US $\$ 44667$. Although part of this discrepancy may be reflected in varying treatment protocols for different countries, this broad range likely also reflects the heterogeneity and uncertainty of inputs included in the cost analyses.

Poor reporting of cost inputs could be related to sources of study funding. Most studies ranked as 'Comprehensive' had funding sources from international organisations or HIC institutions. However, many 'Low'-ranked studies also had funding from HIC institutions and two 
'Comprehensive' studies did not specify any funding source, which suggests that study funding does not necessarily preclude conducting a rigorous economic evaluation.

Finally, only three studies included an analysis that adjusted their results for long-term morbidity and reduced life expectancy due to cancer-related or treatment-related late effects. ${ }^{16} 1830$ Accounting for late effects resulted in decreased cost-effectiveness of the interventions likely due to a reduced number of DALYs averted and higher treatment costs when including costs of managing late effect complications. The impact of late effects of childhood cancer therapy is highly variable and contingent on the type and intensity of treatments applied, with corresponding variability in their impact on cost-effectiveness estimates. Their routine incorporation in future economic analyses of childhood cancer care will be essential to accurate estimation of the societal value of childhood cancer treatment over the life-course.

Despite these limitations, even when restricting our analyses to the most rigorous studies that accounted for key cost inputs and adjusted for late effects, costeffectiveness remained well below internationally accepted GDP-based thresholds. This suggests that even if all omitted expenses were to be included in the less comprehensive studies and late effects were accounted for, LMIC childhood cancer treatment would likely remain consistently very cost-effective.

Given that methodologies including analytic perspective of studies and reported cost inputs varied considerably across studies investigating treatments of the same paediatric cancer, we elected not to conduct a metaanalysis. Improved methodological rigour in future studies will allow opportunity for meta-analysis.

\section{CONCLUSION}

Although paediatric oncology services have often been perceived as too expensive for LMIC health systems, our findings indicate that allocating resources to childhood cancer treatment is a cost-effective investment. While many included studies did not account for key cost inputs and thus underestimated true treatment costs, costs per DALY averted were significantly lower than per capita GDP across diverse LMIC settings, suggesting that even if all relevant inputs are included, LMIC childhood cancer treatment is consistently very cost-effective per WHOCHOICE criteria. While more methodologically rigorous economic evaluations are required, our results can inform the development of LMIC national childhood cancer strategies.

Acknowledgements We thank Thomasin Adams-Webber for her guidance with the literature search.

Contributors Conceptualisation and design: AF, SH, AD, SG. Data analysis and interpretation: AF, SH, VZ, AD, SG. Manuscript writing: AF, SH, AD, SG.

Funding The authors have not declared a specific grant for this research from any funding agency in the public, commercial or not-for-profit sectors.

Competing interests None declared.
Patient consent for publication Not required.

Provenance and peer review Not commissioned; externally peer reviewed.

Data availability statement All data relevant to the study are included in the article or uploaded as supplementary information.

Open access This is an open access article distributed in accordance with the Creative Commons Attribution Non Commercial (CC BY-NC 4.0) license, which permits others to distribute, remix, adapt, build upon this work non-commercially, and license their derivative works on different terms, provided the original work is properly cited, appropriate credit is given, any changes made indicated, and the use is non-commercial. See: http://creativecommons.org/licenses/by-nc/4.0/.

\section{REFERENCES}

1 International Agency for Research on Cancer. Cancer Fact Sheets All cancers: World Health Organization, 2019. Available: http://gco. iarc.fr/today/data/factsheets/cancers/39-All-cancers-fact-sheet.pdf [Accessed 21 May 2019].

2 World Health Organization. Fact Sheets - Cancer: World Health Organization, 2018. Available: https://www.who.int/news-room/factsheets/detail/cancer [Accessed 21 May 2019].

3 National Cancer Institute. Cancer statistics, 2018. Available: https:// www.cancer.gov/about-cancer/understanding/statistics [Accessed 17 Oct 2018].

4 Magrath I, Steliarova-Foucher E, Epelman S, et al. Paediatric cancer in low-income and middle-income countries. Lancet Oncol 2013;14:e104-16.

5 Ward ZJ, Yeh JM, Bhakta N, et al. Estimating the total incidence of global childhood cancer: a simulation-based analysis. Lancet Oncol 2019;20:483-93.

6 The global burden of childhood and adolescent cancer in 2017. An analysis of the global burden of disease study 2017. Lancet Oncol 2019.

7 Hadley LGP, Rouma BS, Saad-Eldin Y. Challenge of pediatric oncology in Africa. Semin Pediatr Surg 2012;21:136-41.

8 Lozano R, Wang H, Foreman KJ, et al. Progress towards millennium development goals 4 and 5 on maternal and child mortality: an updated systematic analysis. The Lancet 2011;378:1139-65.

9 Fadel SA, Boschi-Pinto C, Yu S, et al. Trends in cause-specific mortality among children aged 5-14 years from 2005 to 2016 in India, China, Brazil, and Mexico: an analysis of nationally representative mortality studies. The Lancet 2019;393:1119-27.

10 Smith MA, Seibel NL, Altekruse SF, et al. Outcomes for children and adolescents with cancer: challenges for the twenty-first century. $J$ Clin Oncol 2010;28:2625-34.

11 Rodriguez-Galindo C, Friedrich P, Alcasabas P, et al. Toward the cure of all children with cancer through collaborative efforts: pediatric oncology as a global challenge. JCO 2015;33:3065-73.

12 Eden T, Burns E, Freccero P, et al. Are essential medicines available, reliable and affordable in low-middle income countries? J Cancer Policy 2019;19.

13 Zubizarreta EH, Fidarova E, Healy B, et al. Need for radiotherapy in low and middle income countries - the silent crisis continues. Clin Oncol 2015;27:107-14.

14 Denburg AE, Ramirez A, Pavuluri S, et al. Political priority and pathways to scale-up of childhood cancer care in five nations. PLoS One 2019;14:e0221292.

15 Knaul FM, Gralow JR, Atun R, et al. 1.ii myths and opportunities. North Andover, MA: Closing the Cancer Divide: An Equity Imperative Harvard University Press, 2012.

16 Renner L, Shah S, Bhakta N, et al. Evidence from Ghana indicates that childhood cancer treatment in sub-Saharan Africa is very cost effective: a report from the childhood cancer 2030 network. J Glob Oncol 2018;4:1-9.

17 Bhakta N, Martiniuk ALC, Gupta S, et al. The cost effectiveness of treating paediatric cancer in low-income and middle-income countries: a case-study approach using acute lymphocytic leukaemia in Brazil and Burkitt lymphoma in Malawi. Arch Dis Child 2013;98:155-60.

18 Fuentes-Alabi S, Bhakta N, Vasquez RF, et al. The cost and costeffectiveness of childhood cancer treatment in El Salvador, central America: a report from the childhood cancer 2030 network. Cancer 2018;124:391-7.

19 Hayati $\mathrm{H}$, Kebriaeezadeh A, Ehsani MA, et al. Treatment costs for pediatrics acute lymphoblastic leukemia; comparing clinical expenditures in developed and developing countries: a review article. Int J Pediatrics-Mashhad 2016;4:4033-41. 
20 Russell HV, Panchal J, VonVille H, et al. Economic evaluation of pediatric cancer treatment: a systematic literature review. Pediatrics 2013;131:e273-87.

21 Union for International Cancer Control. Cancer resolution, 2018. Available: https://www.uicc.org/what-we-do/advocacy/globalcommitment/cancer-resolution [Accessed 25 Oct 2018].

22 World Health Organization. Global initiative for childhood cancer, 2018. Available: http://www.who.int/cancer/childhood-cancer/en/ [Accessed 17 Oct 2018].

23 Steliarova-Foucher E, Stiller C, Lacour B, et al. International classification of childhood cancer, third edition. Cancer 2005;103:1457-67.

24 The World Bank. World bank country and lending groups: the world bank group, 2018. Available: https://datahelpdesk.worldbank.org/ knowledgebase/articles/906519-world-bank-country-and-lendinggroups [Accessed 17 Oct 2018].

25 International Monetary Fund. Consumer price index (Cpl), 2019. Available: http://data.imf.org [Accessed 1 Mar 2019].

26 World Health Organization. Health service delivery costs. WHOCHOICE unit cost estimates for service delivery, 2019. Available: https://www.who.int/choice/cost-effectiveness/inputs/health_ service/en/ [Accessed 1 Mar 2019].

27 Swaminathan R, Rama R, Shanta V. Childhood cancers in Chennai, India, 1990-2001: incidence and survival. International Journal of Cancer 2008;122:2607-11.

28 Husereau D, Drummond M, Petrou S, et al. Consolidated Health Economic Evaluation Reporting Standards (CHEERS) - Explanation and Elaboration: A Report of the ISPOR Health Economic Evaluation Publication Guidelines Good Reporting Practices Task Force. Value in Health 2013;16:231-50.

29 Agarwal M, Anchan C, Shah M, et al. Limb salvage surgery for osteosarcoma: effective low-cost treatment. Clin Orthop 2007;459:82-91.

30 Denburg AE, Laher N, Mutyaba I, et al. The cost effectiveness of treating Burkitt lymphoma in Uganda. Cancer 2019;125:1918-28.

31 Gao Y-J, Qian X-W, Lu F-J, et al. Improved outcome for children with non-high risk acute lymphoblastic leukaemia after using an al IC-BFM 2002-based protocol in Shanghai, China. Br J Haematol 2013;160:363-7

32 Garcia M, Alejandra Chicaiza L, Quitian H, et al. Cost-Effectiveness of consolidation treatments for acute myeloid leukemia in highrisk pediatric patients within the Colombian healthcare system. Biomedica 2015;35:549-56.

33 Ghatak N, Trehan A, Bansal D. Financial burden of therapy in families with a child with acute lymphoblastic leukemia: report from North India. Support Care Cancer 2016;24:103-8.

34 LJ G, Li J, Xue HL, et al. Clinical outcome of children with newly diagnosed acute lymphoblastic leukemia treated in a single center in Shanghai, China. Leuk Lymphoma 2008;49:488-94.

35 Hayati $\mathrm{H}$, Kebriaeezadeh A, Ehsani MA, et al. Cost-Utility of protocols of BFM-ALL and UK-ALL for treatment of children with acute lymphoblastic leukemia in Iran. Iranian Journal of Public Health 2018:47:407-12.

36 Hendricks M, Davidson A, Pillay K, et al. Carboplatin-based chemotherapy and surgery: a cost effective treatment strategy for malignant extracranial germ cell tumours in the developing world. Pediatr Blood Cancer 2011;57:172-4.

37 Hesseling PB, Broadhead R, Molyneux E, et al. Malawi pilot study of Burkitt lymphoma treatment. Med Pediatr Oncol 2003;41:532-40.

38 Islam A, Akhter A, Eden T. Cost of treatment for children with acute lymphoblastic leukemia in Bangladesh. $J$ Cancer Policy 2015;6:37-43.

39 Israels T, Paintsil V, Nyirenda D, et al. Improved outcome at end of treatment in the Collaborative Wilms tumour Africa project. Pediatr Blood Cancer 2018;65.

40 Jaime-Perez JC, Heredia-Salazar AC, Cantu-Rodriguez OG, et al. Cost structure and clinical outcome of a stem cell transplantation program in a developing country: the experience in northeast Mexico. Oncologist 2015;20:386-92.

41 Jasso-Gutierrez L, Acosta ED, Rodriguez Ortega EE, et al. Estimation of chemotherapy costs applying the full protocol of children with acute lymphoblastic leukemia or Hodgkin's lymphoma: case study. Boletin Medico del Hospital Infantil de Mexico 2012;69:10-21.

42 Jayaraman D, Uppuluri R, Swaminathan VV, et al. Affordable and safe health care for all children: lessons learned from the use of PEG-asparaginase in a developing country. Indian J Med Paediatr Oncol 2017;38:398-400.
$43 \mathrm{Ji}$ X, Xuan Y, Li J, et al. Direct costs for retinoblastoma treatment during the first year of comprehensive therapy in China. $J$ Pediatr Ophthalmol Strabismus 2012;49:353-8.

44 Kanyamuhunga A, Tuyisenge L, Stefan DC. Treating childhood cancer in Rwanda: the nephroblastoma example. Pan Afr Med J 2015;21.

45 Liu Y, Chen J, Tang J, et al. Cost of childhood acute lymphoblastic leukemia care in Shanghai, China. Pediatr Blood Cancer 2009;53:557-62.

46 Luo X-Q, Ke Z-Y, Guan X-Q, et al. The comparison of outcome and cost of three protocols for childhood non-high risk acute lymphoblastic leukemia in China. Pediatr Blood Cancer 2008;51:204-9.

47 Luo X-Q, Ke Z-Y, Huang L-B, et al. Improved outcome for Chinese children with acute promyelocytic leukemia: a comparison of two protocols. Pediatr Blood Cancer 2009;53:325-8.

48 Meremikwu MM, Ehiri JE, Nkanga DG, et al. Socioeconomic constraints to effective management of Burkitt's lymphoma in southeastern Nigeria. Trop Med Int Health 2005;10:92-8.

49 Neal C, Rusangwa C, Borg R, et al. Cost of treating pediatric cancer at the Butaro cancer center of excellence in Rwanda. J Glob Oncol 2018;4:1-7.

50 Paintsil V, David H, Kambugu J, et al. The Collaborative Wilms tumour Africa project; baseline evaluation of Wilms tumour treatment and outcome in eight Institutes in sub-Saharan Africa. Eur $J$ Cancer 2015;51:84-91.

51 Rocha-Garcia A, Hernandez-Pena P, Ruiz-Velazco S, et al. OutOf-Pocket expenditures during hospitalization of young leukemia patients with state medical insurance in two Mexican hospitals. Salud Publica De Mexico 2003;45:285-92.

52 Stefan DC, Stones D. How much does it cost to treat children with Hodgkin lymphoma in Africa? Leuk Lymphoma 2009;50:196-9.

53 Traoré F, Coze C, Atteby J-J, et al. Cyclophosphamide monotherapy in children with Burkitt lymphoma: a study from the FrenchAfrican pediatric Oncology Group (GFAOP). Pediatr Blood Cancer 2011;56:70-6.

54 Waddell KM, Kagame K, Ndamira A, et al. Improving survival of retinoblastoma in Uganda. Br J Ophthalmol 2015;99:937-42.

55 JJA Y, Kabesha TA, Budiongo AN, et al. Retinoblastoma in subSaharan Africa: case studies of the Republic of Cote d'Ivoire and the Democratic Republic of the Congo. J Glob Oncol 2018;4:1-8.

56 Woods B, Revill P, Sculpher M, et al. Country-Level costeffectiveness thresholds: initial estimates and the need for further research. Working papers. Centre for Health Economics, University of York, 2015: 109cherp.

57 Stenberg K, Sweeny K, Axelson H, et al. Returns on Investment in the Continuum of Care for Reproductive, Maternal, Newborn, and Child Health. In: Black RE, Laxminarayan R, Temmerman M, et al, eds. Reproductive, maternal, newborn, and child health: disease control priorities, third edition, volume 2. Washington, DC: The International Bank for Reconstruction and Development / The World Bank (c) 2016 International Bank for Reconstruction and Development / The World Bank, 2016.

58 Horton S, Levin C. Cost-Effectiveness of interventions for reproductive, maternal, neonatal, and child health in: black $R$, editor. reproductive, maternal, newborn, and child health: disease control priorities. 2. third ED. Washington, DC: The International Bank for Reconstruction and Development / The World Bank, 2016.

59 Horton S, Gelband H, Jamison D, et al. Supplementary table 1. details of interventions included in figures 1, 2, 3 and 4, ordered by increasing cost per DALY averted. in ranking 93 health interventions for low- and middle-income countries by cost-effectiveness. PloS one 2017;12:e0182951.

60 Atun R, Bhakta N, Denburg A, et al. Lancet oncology Commission for sustainable care for children with cancer. Lancet Oncology In press.

61 Gupta S, Yeh S, Martiniuk A, et al. The magnitude and predictors of abandonment of therapy in paediatric acute leukaemia in middleincome countries: a systematic review and meta-analysis. Eur $J$ Cancer 2013;49:2555-64.

62 Gupta S, Rivera-Luna R, Ribeiro RC, et al. Pediatric oncology as the next global child health priority: the need for national childhood cancer strategies in low- and middle-income countries. PLoS Med 2014:11:e1001656.

63 Sabik LM, Lie RK. Priority setting in health care: lessons from the experiences of eight countries. Int J Equity Health 2008;7:4.

64 Horton S, Gupta S. Costing methods applied to cancer. J Glob Oncol 2019;5:1-3. 